\title{
Clinical and cost-effectiveness of social recovery therapy for the prevention and treatment of long-term social disability among young people with emerging severe mental illness (PRODIGY): randomised controlled trial
}

Clio Berry, Joanne Hodgekins, Paul French, Tim Clarke, Lee Shepstone, Garry Barton, Robin Banerjee, Rory Byrne, Rick Fraser, Kelly Grant, Kathryn Greenwood, Caitlin Notley, Sophie Parker, Jon Wilson, Alison R. Yung and David Fowler

\section{Background}

Young people with social disability and severe and complex mental health problems have poor outcomes, frequently struggling with treatment access and engagement. Outcomes may be improved by enhancing care and providing targeted psychological or psychosocial intervention.

\section{Aims}

We aimed to test the hypothesis that adding social recovery therapy (SRT) to enhanced standard care (ESC) would improve social recovery compared with ESC alone.

\section{Method}

A pragmatic, assessor-masked, randomised controlled trial (PRODIGY: ISRCTN47998710) was conducted in three UK centres. Participants $(n=270)$ were aged $16-25$ years, with persistent social disability, defined as under 30 hours of structured activity per week, social impairment for at least 6 months and severe and complex mental health problems. Participants were randomised to ESC alone or SRT plus ESC. SRT was an individual psychosocial therapy delivered over 9 months. The primary outcome was time spent in structured activity 15 months post-randomisation.

\section{Results}

We randomised 132 participants to SRT plus ESC and 138 to ESC alone. Mean weekly hours in structured activity at 15 months increased by $11.1 \mathrm{~h}$ for SRT plus ESC (mean 22.4, s.d. = 21.4) and
16.6 h for ESC alone (mean 27.7, s.d. = 26.5). There was no significant difference between arms; treatment effect was -4.44 (95\% $\mathrm{Cl}-10.19$ to $1.31, P=0.13$ ). Missingness was consistently greater in the ESC alone arm.

\section{Conclusions}

We found no evidence for the superiority of SRT as an adjunct to ESC. Participants in both arms made large, clinically significant improvements on all outcomes. When providing comprehensive evidence-based standard care, there are no additional gains by providing specialised SRT. Optimising standard care to ensure targeted delivery of existing interventions may further improve outcomes.

\section{Keywords}

Social functioning; psychosocial interventions; depressive disorders; anxiety disorders; cognitive-behavioural therapies.

\section{Copyright and usage}

(c) The Author(s), 2022. Published by Cambridge University Press on behalf of the Royal College of Psychiatrists. This is an Open Access article, distributed under the terms of the Creative commons Attribution licence (https://creativecommons.org/ licenses/by/4.0/), which permits unrestricted re-use, distribution, and reproduction in any medium, provided the original work is properly cited.
The literature on psychological or psychosocial interventions for young people with social disability and severe and complex mental health problems is sparse. ${ }^{1-6}$ Of the four studies that we identified, only one, of a brief group workshop-based intervention targeting clinical symptoms and resilience, appeared significantly effective; ${ }^{6}$ however, the study had no control group and the student sample was not a clinical sample.

Three-quarters of socially disabling long-term mental health problems begin in adolescence. ${ }^{7}$ Social disability is observable before the onset of mental health problems and if it is ongoing, transition to long-term problems of diagnostic severity is more likely. ${ }^{2,8}$ Social disability can be defined as low time spent in structured activity. Young people without mental health problems spend 64 hours a week ('weekly hours') on average in structured activity, with under $30 \mathrm{~h}$ representing serious social disability and under $15 \mathrm{~h}$ severe social disability. ${ }^{9}$ Structured activity includes paid and voluntary employment, education, caring, sports and structured leisure. ${ }^{9}$ Young people at the greatest risk of long-term social disability present with emerging social withdrawal, emotional and behavioural problems, subthreshold psychosis and risky behaviours such as alcohol and drug use. ${ }^{7}$ This group is extremely vulnerable and their problems, if untreated, have severe, potentially lifelong personal and economic costs. ${ }^{7,10}$ This group is neglected in research and clinical care, with under $25 \%$ of young people with a diagnosable mental health problem gaining access to specialist mental health services. ${ }^{11}$ The significant social withdrawal makes engagement very challenging ${ }^{2,5}$ and standard interventions may be inaccessible and ineffective. Recent reviews demonstrate that cognitive-behavioural therapy (CBT) has only small or short-term effects on social outcomes for people at risk of psychosis or with schizophrenia. $^{12,13}$ Youth community services, for example serving young people not in employment, education or training (NEET), often provide excellent vocational support but lack the requisite mental health specialism for this complex group. ${ }^{14}$ Vocational interventions delivered in mental health services, such as individual placement and support, are effective in increasing paid employment for people with severe mental health problems, but do not target broader clinical and social outcomes. ${ }^{15}$ Early intervention in psychosis (EIP) services assertively engage people with severe and complex mental health problems in specialist mental health and socio-vocational support, but are only accessible with a confirmed or imminent first episode of psychosis, thus excluding people with 
non-psychotic yet severe and complex mental health problems. Furthermore, most people accessing EIP still experience persistent social disability 1 year later, ${ }^{16}$ and non-psychosis specific youth mental health services, where these exist, effect reliable change for only a minority. ${ }^{4}$

We developed social recovery therapy (SRT), a specialist psychosocial intervention combining CBT, assertive outreach and multisystemic principles to treat social disability in the context of severe and complex mental health problems. ${ }^{17}$ The clinical and cost-effectiveness of SRT has been demonstrated in psychosis. ${ }^{18,19}$ The SUPEREDEN3 trial $^{19}$ showed that adding SRT to EIP produced large and significant improvements in structured activity and psychotic and mood symptoms. We hypothesised that providing SRT to a younger group with emerging severe problems would create early gains in social and clinical recovery, interrupting potentially lifelong trajectories of disability. We conducted the PRODIGY trial to test the benefit of adding SRT to optimised standard care for young people with severe and complex mental health problems who had not experienced an episode of psychosis. We conducted a qualitative process evaluation alongside the trial. ${ }^{20,21}$ Here we present the trial outcomes. We aimed to test the primary hypothesis that SRT as an adjunct to enhanced standard care (ESC) would be superior to ESC alone in improving weekly hours in structured activity at 15 months post-randomisation. ${ }^{1}$ Secondary hypotheses were that the addition of SRT would lead to greater improvements in psychotic, mood and general symptoms at 9 and 15 months, and that improvements would persist until 24 months post-randomisation. ${ }^{1}$

\section{Method}

\section{Study design}

This was a pragmatic, multi-centre, single- (assessor-) blind, randomised controlled trial comparing the clinical and cost-effectiveness of social recovery therapy (SRT) and enhanced standard care (ESC) with ESC alone. The intervention period lasted 9 months post-randomisation. Outcomes were assessed at 9 and 15 months post-randomisation, with limited assessment of longer-term outcomes at 24 months post-randomisation.

\section{Participants}

The target sample size was 270 participants, 135 in each arm. ${ }^{1}$ This target was calculated using a minimal clinically important effect size of 0.4 standard deviations in weekly structured activity hours, with $90 \%$ statistical power, two-sided 5\% significance level, and allowing $20 \%$ attrition. Inclusion criteria were: aged 16-25 years; persistent social disability; severe and complex mental health problems. Persistent social disability was operationalised as $<30$ weekly hours of structured activity, with history of social impairment for at least 6 months. The presence of severe and complex mental health problems was operationalised as meeting at risk mental state for psychosis (ARMS) criteria and/or scoring $\leq 50$ on the Global Assessment of Functioning Scale (indicating at least serious symptoms and/or serious impairment in social, occupational or school functioning) with persistence of at least moderate symptoms for at least 6 months. Exclusion criteria were: active psychotic symptoms or history of psychosis measured using Comprehensive Assessment of At Risk Mental States (CAARMS) criteria; severe intellectual disability; non-English speaking; disease or physical problems likely to undermine participation. Participants were recruited from primary and secondary youth and adult National Health Service (NHS) mental health services, and third-sector, youth, employment, and educational support organisations.

\section{Randomisation and masking}

Following baseline assessment, participants were randomly allocated 1:1 to receive SRT plus ESC or ESC alone. Remote randomisation was performed by Norwich Clinical Trials Unit (NCTU) using pre-determined lists with randomly distributed block sizes of 4 or 6. Randomisation was stratified by age (16-19 and 20-25 years), site (Sussex, East Anglia or Manchester), social disability severity (low functioning: 16-30 weekly hours of structured activity; versus very low functioning: $0-15$ weekly hours) and whether ARMS criteria were met. The allocation sequence was hidden from all system users. A remote web-based system notified the allocation to NCTU and nominated trial staff.

Research assistants collecting baseline and follow-up data were masked to intervention allocation. Masking was maintained by restricted access to the data management system and in-office precautions. Thirty-one unmaskings occurred during the trial, in which the assessor became aware of whether a participant had or had not received SRT in addition to ESC. All unmaskings were successfully managed by reallocating outcome data collection to another masked assessor.

\section{Procedures}

Ethical approval was provided by the East of England Research Ethics Committee (12/EE/0311) and the Preston Research Ethics Committee North West (15/NW/0590). Potentially eligible participants were approached by their usual care provider, who (with permission) shared contact details with the research team. Potential participants were provided with written and verbal information describing trial involvement, and were invited to provide written informed consent and complete a screening assessment. Eligibility was confirmed through trial management group review. Eligible participants completed a baseline assessment and were randomised.

All participants received enhanced standard care (ESC). ESC involved services already received or offered throughout the trial, including psychological therapies where applicable. We anticipated that standard care might be limited and inaccessible. We enhanced standard care by offering a comprehensive assessment report, detailing current mental health and social functioning, to all participants and providers at trial entry and follow-up assessments. Providers were additionally given a comprehensive best practice guide, detailing local services and organisations to which participants could be referred. Providers were encouraged to offer optimal clinical evidence-based services according to National Institute for Health and Care Excellence (NICE) guidelines. All three participating research sites were centres of excellence for youth mental health and psychosis.

Participants randomised to social recovery therapy (SRT) plus ESC additionally received up to 9 months of individual SRT sessions. SRT (www.socialrecoverytherapy.co.uk) incorporates assertive outreach, multisystemic and CBT techniques. Compared with traditional CBT, SRT focuses on assessment and formulation of barriers to social recovery, with a particular focus on using behavioural work and engaging with structured activity providers and others in the surrounding system to maintain social recovery. SRT was delivered according to the therapy manual. ${ }^{17}$ First, efforts were made to initiate engagement and develop a positive therapeutic relationship, with assessment of goals and barriers used to derive a social recovery formulation. Sessions were intended to be weekly at outset, reducing to fortnightly or monthly towards the end of the intervention period. Behavioural assessment during activities in the community informed the assessment and formulation. Following this, therapy focused on increasing time spent in structured activities linked to personal goals. Cognitive techniques promoted hopefulness and addressed negative beliefs about the self and others. Multi-layered 
behavioural experiments were used to manage symptoms while participants were engaging in structured activity; examples are provided in the therapy manual. ${ }^{17}$ SRT appears to improve structured activity through augmenting positive self-beliefs. ${ }^{22}$ The SRT therapists were clinical psychologists, occupational therapists, or mental health nurses with CBT accreditation. All the therapists received training in SRT and regular expert individual and peer supervision. Three raters measured SRT adherence with our developed checklist ${ }^{23}$ using session audiotapes and therapist notes. Interrater reliability was excellent; Krippendorff's $\alpha=0.9$ (95\% CI 0.87-0.98). A full SRT dose was defined as six or more sessions, with a social recovery assessment and formulation, and two or more pieces of behavioural work in the community with the therapist. Adherence data showed that $64 \%$ of sessions included behavioural work, $36 \%$ of which included a behavioural experiment. Of the face-to-face sessions, $53 \%$ were delivered in the participant's home, $24 \%$ in the community (e.g. meeting in a city centre), $10 \%$ in an educational setting (e.g. meeting at college) and 13\% in a clinic setting. Therapist competence was rated using session audiotapes. Competence was defined as a total score $\geq 36$ on the Revised Cognitive Therapy Scale (CTS-R). ${ }^{24}$ The CTS-R measures the therapist's general competence in delivering cognitive therapy, i.e. using therapeutic skills and techniques, such as collaboration and feedback, to support cognitive change.

\section{Outcomes}

Participants were assessed at baseline, 9 and 15 months post-randomisation, with limited assessment of maintenance at 24 months. Research assistants used flexible, assertive engagement to facilitate involvement, conducting assessments mainly in participants' homes. High interrater reliability for assessor-rated measures was ensured through regular supervision and training.

The primary outcome was weekly hours spent in structured activity. ${ }^{9}$ Using a structured interview derived from the Office for National Statistics Time Use Survey, ${ }^{25}$ time spent over the past month in structured activity was captured. Past month activity was divided to reflect average weekly hours in constructive economic (paid and voluntary employment, childcare, housework and chores) and structured activity (constructive economic activity plus sports and leisure). The primary end-point was 15 months post-randomisation.

Levels of attenuated psychotic symptoms were measured using the Comprehensive Assessment of At Risk Mental States (CAARMS). Derived outcomes included transition to psychosis and CAARMS symptom severity and symptom distress scores. ${ }^{26}$ Negative symptoms were assessed using the Scale for Assessment of Negative Symptoms (SANS). General psychopathology was measured as change in mood, anxiety, somatoform and eating disorders using the Structured Clinical Interview for DSMIV, and additionally with the self-report Beck Depression Inventory II (BDI-II), Social Interaction Anxiety Scale (SIAS), and the assessor-rated Global Assessment of Functioning (GAF), Global Assessment of Symptoms (GAS) and Social and Occupational Functioning Scale (SOFAS). Putative mediators were assessed using the Acceptance and Action Questionnaire II (AAQ-II), Meaning in Life Questionnaire (MLQ), Trait Hope Scale (THS), Brief Core Schema Scales (BCSS) and Schizotypal Symptoms Inventory (SSI). Putative moderators were verbal memory, captured using the Logical Memory I subtest of the Wechsler Memory Scale, Third Edition (LMS), and verbal fluency, captured using the Controlled Oral Word Association Test (COWAT). Other outcomes were scores on the Beck Hopelessness Scale (BHS), Alcohol Use Disorders Identification Test (AUDIT) and Drug Use Disorders Identification Test
(DUDIT). Health economic outcomes were NHS and personal social service use, captured using the Health Service Resource Use Questionnaire adapted from the Client Service Receipt Inventory (CSRI), and health-related quality of life, captured using the EuroQol EQ-5D-3L. Adverse events were recorded throughout the trial and reported to trial oversight committees.

\section{Statistical analysis}

Primary and secondary hypotheses were pre-registered (ISRCTN47998710;29 November 2012) and published in the protocol. ${ }^{1}$ A detailed statistical analysis plan was agreed between the chief investigators, trial manager, trial statistician and health economist on 4 July 2018, prior to analysis. The primary analysis was intention to treat (ITT) comparing SRT plus ESC with ESC alone on weekly structured activity hours at 15 months post-randomisation. A per protocol analysis was also conducted, involving participants who received full-dose SRT. All analyses were conducted by the trial statistician and health economist. All hypothesis testing was conducted

\begin{tabular}{|c|c|c|}
\hline Characteristic & $\begin{array}{c}\text { SRT + ESC } \\
(n=138)\end{array}$ & $\begin{array}{c}\text { ESC alone } \\
(n=132)\end{array}$ \\
\hline \multicolumn{3}{|l|}{ Age group, years: $n$ (\%) } \\
\hline $16-19$ & $79(57)$ & $75(57)$ \\
\hline $20-25$ & $59(43)$ & $57(43)$ \\
\hline \multicolumn{3}{|l|}{ Age, years } \\
\hline Mean (s.d.) & $20.1(2.5)$ & $20.0(2.7)$ \\
\hline Missing, $n$ & 6 & 5 \\
\hline \multicolumn{3}{|l|}{ Gender, $n$ (\%) } \\
\hline Female & $54(39)$ & $66(50)$ \\
\hline Male & $84(61)$ & $66(50)$ \\
\hline \multicolumn{3}{|l|}{ Ethnicity, $n$ (\%) } \\
\hline White & $127(92)$ & $114(86)$ \\
\hline Black and minority ethnic & $11(8.0)$ & $18(14)$ \\
\hline \multicolumn{3}{|l|}{ Marital status, $n(\%)$} \\
\hline Partner & $18(13)$ & $17(13)$ \\
\hline Separated & $2(1.5)$ & 0 \\
\hline Single & $118(86)$ & $115(87)$ \\
\hline \multicolumn{3}{|l|}{ Employment status, $n$ (\%) } \\
\hline Paid work & $5(3.6)$ & $6(4.6)$ \\
\hline Voluntary work & $3(2.2)$ & $4(3.0)$ \\
\hline Student & $34(25)$ & $31(24)$ \\
\hline Unemployed & $95(69)$ & $91(69)$ \\
\hline Missing & 1 & 0 \\
\hline \multicolumn{3}{|l|}{ Sexual orientation, $n$ (\%) } \\
\hline Heterosexual & $98(74)$ & $107(82)$ \\
\hline Homosexual & $6(4.5)$ & $6(4.6)$ \\
\hline Bisexual & $16(12.1)$ & $13(9.9)$ \\
\hline Unsure & $6(4.5)$ & $1(0.8)$ \\
\hline Other & $6(4.5)$ & $4(3.1)$ \\
\hline Missing & 6 & 1 \\
\hline \multicolumn{3}{|l|}{ Accommodation, $n(\%)$} \\
\hline Accommodation with support & $8(5.9)$ & $4(3.0)$ \\
\hline Homeless/temporary accommodation & $5(3.7)$ & $7(5.3)$ \\
\hline Mobile accommodation & 0 & $1(0.8)$ \\
\hline Owner occupied & $48(36)$ & $41(31)$ \\
\hline $\begin{array}{l}\text { Rented (local authority/ housing } \\
\text { association) }\end{array}$ & $45(33)$ & $55(42)$ \\
\hline Rented (private) & $29(22)$ & $24(18)$ \\
\hline Missing & 3 & 0 \\
\hline \multicolumn{3}{|l|}{ Social disability, $n(\%)$} \\
\hline Low functioning & $40(29)$ & $40(30)$ \\
\hline Very low functioning & $98(71)$ & $92(70)$ \\
\hline \multicolumn{3}{|l|}{ ARMS status, $n(\%)$} \\
\hline At risk & $69(50)$ & $64(49)$ \\
\hline Not at risk & $69(50)$ & $68(52)$ \\
\hline
\end{tabular}


using a two-sided significance level of 5\%, with corresponding 95\% confidence intervals.

We anticipated that the primary outcome might have positive skew and require a logarithmic transformation. Assuming normal (or transformed to normal) distribution, a general linear model was constructed for primary and then secondary analyses. For the primary outcome analyses, baseline logical memory and verbal fluency were included as prognostic variables, alongside stratification variables (site as a random factor) and allocation. Secondary outcomes were analysed using an analogous approach: a linear model with appropriate link for the outcome (e.g. logistic regression for binary outcomes) and including stratification variables, predefined prognostic variables and allocation. Available baseline values of the outcome were included. Planned moderation analysis considered the impact on the intervention effect of baseline social disability (low or very low functioning), ARMS status, logical memory and verbal fluency. One addition was made to the statistical analysis plan after publication of the registered trial protocol: we added models that excluded time spent in childcare, as this variable showed an imbalance towards females that considerably inflates structured activity. Both model types are presented here. Analyses were conducted using SAS for Windows (version 9.4).

Health economic outcomes were analysed using a within-trial cost-utility ITT approach, where costs (at 2017-2018 levels) and benefits were estimated over 24 months, with a $3.5 \%$ discount in the second year. SRT costs included therapy training, supervision and delivery. ESC costs were derived from reported service contacts. Total quality-adjusted life-year (QALY) scores were estimated using EQ-5D-3L data. Regression was used to estimate (separately) the mean incremental cost and mean QALY gain. The incremental cost-effectiveness ratio (ICER) (mean incremental cost/mean QALY gain) was estimated, with value for money corresponding to the cost-effectiveness threshold of $£ 20000$ per QALY. The level of uncertainty, according to the cost-effectiveness acceptability curve, was assessed at the same cost-effectiveness threshold value. Multiple imputation was used in the base-case analysis.

\section{Results}

One hundred participants were recruited in an internal pilot, from 1 January 2013 to 1 February 2014 ( $n=50$ in East Anglia and $n=50$ Manchester). The remaining 170 participants $(n=57$ in Sussex, $n=59$ in East Anglia and $n=54$ in Manchester) were recruited during an extension phase between 1 September 2015 and 31 May 2017. In total, 270 participants were randomised to receive social recovery therapy (SRT) plus enhanced standard care (ESC) $(n=138)$ or ESC alone $(n=132)$. Baseline characteristics were similar across groups (Table 1). The ESC alone group was evenly balanced between sexes, but the SRT plus ESC group overrepresented males. Baseline data (Table 2) reflect severe social disability and psychiatric health symptoms. Most participants met diagnostic criteria for major depression and almost half for current social phobia.

Participants allocated to SRT received a mean of 16.77 therapy sessions, with a range of 0 to 33. SRT adherence ratings demonstrated that $91(66 \%)$ participants received the full dose, 23 $(17 \%)$ a partial dose and $24(17 \%)$ no dose $(n=2$ attended zero sessions). Seventy-five session tapes, at least one per therapist, were rated for competence. The mean CTS-R score was 47.24, with 97\% of sessions rated above the competence threshold of 36 . ESC provision was substantial in both arms. Over $80 \%$ of participants in both arms accessed NHS mental health services at trial entry, which continued for more than two-thirds throughout the intervention period, and for more than half throughout the trial. Between each assessment point, a mean of $38 \%$ of ESC and 39\% of SRT participants saw a care coordinator or case manager on average $9-11$ times, and $33 \%$ of ESC and $25 \%$ of SRT participants saw a psychological therapist, on average for 7-12 sessions. An average of $66 \%$ of ESC and $72 \%$ of SRT participants saw their general practitioner on average 3-5 times between each assessment point. An average of $19 \%$ of ESC and $14 \%$ of SRT participants saw a psychiatrist between each assessment point, on average 1-3 times. An average of $51 \%$ ESC and $54 \%$ of SRT

\begin{tabular}{|c|c|c|c|c|c|c|c|c|}
\hline \multirow[b]{2}{*}{ Outcome } & \multicolumn{2}{|c|}{ Baseline } & \multicolumn{2}{|c|}{9 months } & \multicolumn{2}{|c|}{15 months } & \multicolumn{2}{|c|}{24 months } \\
\hline & $\mathrm{SRT}+\mathrm{ESC}$ & ESC alone & $\mathrm{SRT}+\mathrm{ESC}$ & ESC alone & $\mathrm{SRT}+\mathrm{ESC}$ & ESC alone & $\mathrm{SRT}+\mathrm{ESC}$ & ESC alone \\
\hline \multicolumn{9}{|c|}{ Primary outcomes, hours per week: mean (s.d.) } \\
\hline Structured activity & $11.3(8.0)$ & $11.3(8.6)$ & $21.4(16.6)$ & $22.3(19.3)$ & $22.4(21.4)$ & $27.7(26.5)$ & $24.3(18.9)$ & $32.4(28.7)$ \\
\hline Structured activity without childcare & $11.0(7.8)$ & $11.2(8.6)$ & $20.3(14.7)$ & $22.2(19.3)$ & $21.1(18.1)$ & $24.9(20.4)$ & $23.8(18.9)$ & $26.6(20.4)$ \\
\hline Constructive economic activity & $8.6(7.1)$ & $8.1(7.0)$ & $15.7(14.3)$ & $16.6(15.9)$ & $17.4(19.9)$ & $22.0(24.5)$ & $18.6(16.7)$ & $27.4(28.0)$ \\
\hline \multicolumn{9}{|l|}{ Secondary outcomes } \\
\hline Transition to psychosis, $n(\%)$ & - & - & $12(9.8)$ & $8(7.5)$ & $6(5.6)$ & $1(1.1)$ & $3(3.1)$ & $2(2.5)$ \\
\hline CAARMS symptom severity, mean (s.d.) & $26.2(16.5)$ & $26.1(15.9)$ & $28.4(21.5)$ & $27.1(18.7)$ & $23.4(21.0)$ & $24.3(18.9)$ & $20.4(21.3)$ & $20.2(19.4)$ \\
\hline CAARMS symptom distress, mean (s.d.) & $52.5(27.0)$ & $52.1(23.1)$ & $47.5(27.5)$ & $44.7(24.3)$ & $43.4(28.1)$ & $39.7(25.8)$ & $42.8(26.6)$ & $31.0(26.5)$ \\
\hline Current major depressive episode, $n$ (\%) & $72(52.2)$ & 65 (49.2) & $26(21)$ & $33(29.2)$ & $35(29)$ & $26(25.7)$ & $24(22.6)$ & $18(21.7)$ \\
\hline Major depressive disorder, $n(\%)$ & $95(68.8)$ & $93(70.5)$ & $66(52.8)$ & $55(49.6)$ & $58(47.5)$ & $43(42.2)$ & $45(42.5)$ & $28(33.7)$ \\
\hline Current social phobia, $n(\%)$ & $62(?)$ & $54(40.9)$ & $22(40)^{\mathrm{b}}$ & $24(53.3)^{b}$ & $28(52)^{\mathrm{b}}$ & $16(39.0)^{b}$ & $27(59)^{\mathrm{b}}$ & $13(36.1)$ \\
\hline Current generalised anxiety disorder, $n(\%)$ & $36(?)$ & 44 (33.3) & $11(33)$ & $13(41)$ & $10(30.3)$ & $5(46.5)$ & $13(48)$ & 11 (42\%) \\
\hline BDI-II, mean (s.d.) & $30.4(12.8)$ & $30.3(12.4)$ & $18.6(15.4)$ & $19.9(13.7)$ & $19.2(15.7)$ & $19.4(14.9)$ & $18.0(15.7)$ & $17.5(14.8)$ \\
\hline SIAS, mean (s.d.) & $52.1(14.1)$ & $48.1(16.1)$ & $44.1(16.9)$ & $44.0(15.6)$ & $43.1(17.7)$ & $42.2(17.7)$ & $43.9(17.6)$ & $41.3(17.4)$ \\
\hline GAF, mean (s.d.) & $37.9(5.6)$ & $38.2(5.5)$ & $49.7(15.8)$ & $48.6(14.9)$ & $50.8(18.0)$ & $51.9(17.4)$ & $50.3(17.2)$ & $53.4(16.2)$ \\
\hline GAS, mean (s.d.) & $43.1(7.3)$ & $43.2(7.5)$ & $52.2(15.2)$ & $51.2(14.1)$ & $54.2(16.0)$ & $55.6(17.9)$ & $53.3(17.6)$ & $56.6(16.6)$ \\
\hline SOFAS, mean (s.d.) & $41.6(7.6)$ & $43.3(7.0)$ & $51.7(15.5)$ & $53.4(16.5)$ & $54.6(17.3)$ & $55.8(19.4)$ & $53.3(18.1)$ & $57.4(19.4)$ \\
\hline \multicolumn{9}{|l|}{ Other outcomes, mean (s.d.) } \\
\hline $\mathrm{BHS}$ & $13.4(5.8)$ & $12.7(5.2)$ & $9.2(6.2)$ & $9.1(5.9)$ & $9.5(6.1)$ & $9.5(6.4)$ & $9.6(6.1)$ & $7.8(6.2)$ \\
\hline AUDIT & $5.0(6.3)$ & $5.2(6.3)$ & $4.6(6.6)$ & $4.4(5.1)$ & $4.6(6.0)$ & $4.5(6.0)$ & $3.5(4.3)$ & 3.7 (3.3) \\
\hline DUDIT & $3.6(7.2)$ & $3.9(7.8)$ & $3.1(6.6)$ & $3.8(7.8)$ & $2.6(5.7)$ & $3.4(7.9)$ & $2.1(6.0)$ & $3.3(7.3)$ \\
\hline
\end{tabular}




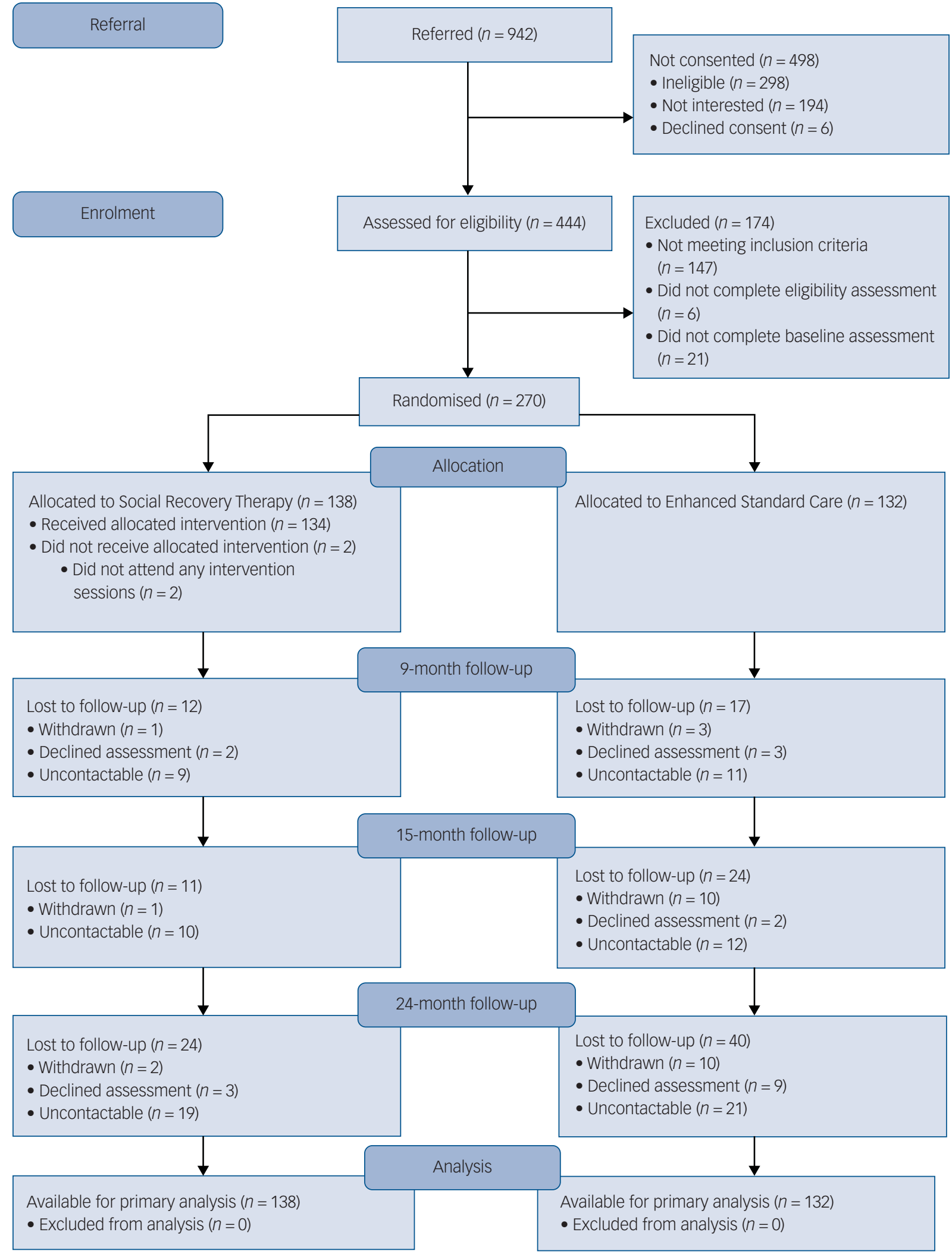

Fig. 1 Participant flow diagram.

participants reported antidepressant use, and a substantial minority reported use of other medications, between each assessment point. One-third of participants in both arms reported support for employment over the trial for a mean total of 11.3 (ESC: range $0.3-76.5 \mathrm{~h}$ ) and $75.3 \mathrm{~h}$ (SRT: range $0.5-842.4 \mathrm{~h}$ ). Over
$40 \%$ of ESC participants and $32 \%$ of SRT participants reported other contact with youth, financial, educational, social, housing, statutory and telephone support services over the trial, for a mean total of 14.6 (ESC: range $0.2-225.0 \mathrm{~h}$ ) and $7.9 \mathrm{~h}$ (SRT: range $0.2-74.0 \mathrm{~h})$. 
Table 3 Pre-specified primary outcome analysis, and secondary time use outcome analysis, using intention-to-treat population and per protocol population $^{\mathrm{a}}$

\begin{tabular}{|c|c|c|c|c|c|c|}
\hline \multirow[b]{2}{*}{ Outcome and time point } & \multicolumn{2}{|c|}{ Intention-to-treat population } & \multicolumn{2}{|c|}{ Missing data } & \multicolumn{2}{|c|}{ Per protocol population } \\
\hline & $\begin{array}{c}\text { Adjusted mean } \\
\text { difference }(95 \% \mathrm{Cl}) \text {, } \\
\text { (Cohen's } d)\end{array}$ & $\begin{array}{l}\text { Intervention } v \text {. control, } \\
\text { log-transformed } P\end{array}$ & $\begin{array}{c}\mathrm{SRT}+\mathrm{ESC} \\
(n=138) \\
n(\%)\end{array}$ & $\begin{array}{c}\text { ESC alone } \\
(n=132) \\
n(\%)\end{array}$ & $\begin{array}{c}\text { Adjusted mean } \\
\text { difference }(95 \% \mathrm{Cl})\end{array}$ & $\begin{array}{c}P \text {-value intervention } \\
\text { v. control (log } \\
\text { transformed } P \text {-value) }\end{array}$ \\
\hline \multicolumn{7}{|l|}{ Primary outcome } \\
\hline $\begin{array}{l}\text { Structured activity at } \\
9 \text { months }\end{array}$ & $\begin{array}{c}-0.90(-5.02 \text { to } 3.21) \\
(-0.05)\end{array}$ & $0.67(0.50)$ & $12(8.7)$ & $17(12.9)$ & $-1.23(-5.71$ to 3.25$)$ & $0.59(0.45)$ \\
\hline $\begin{array}{l}\text { Structured activity at } \\
15 \text { months }\end{array}$ & $\begin{array}{c}-4.44(-10.19 \text { to } 1.31) \\
(-0.22)\end{array}$ & $0.13(0.29)$ & $11(8.0)$ & $24(18.2)$ & $-7.00(-13.22$ to -0.78$)$ & $0.03(0.19)$ \\
\hline $\begin{array}{l}\text { Structured activity at } \\
24 \text { months }\end{array}$ & $\begin{array}{c}-7.51(-13.91 \text { to } \\
-1.12),(-0.33)\end{array}$ & $0.02(0.099)$ & $25(18.1)$ & $40(30.3)$ & $-8.75(-16.07$ to -1.42$)$ & $0.02(0.059)$ \\
\hline $\begin{array}{l}\text { Structured activity } \\
\text { without childcare at } \\
9 \text { months }\end{array}$ & $\begin{array}{c}-1.71(-5.67 \text { to } 2.26) \\
(-0.11)\end{array}$ & $0.40(0.57)$ & $12(8.7)$ & $17(12.9)$ & -2.04 (-6.41 to 2.34$)$ & $0.36(0.52)$ \\
\hline $\begin{array}{l}\text { Structured activity } \\
\text { without childcare at } \\
15 \text { months }\end{array}$ & $\begin{array}{c}-2.98(-7.49 \text { to } 1.53) \\
(-0.20)\end{array}$ & $0.19(0.46)$ & $11(8.0)$ & $24(18.2)$ & $-4.99(-9.89$ to -0.08$)$ & $0.05(0.31)$ \\
\hline $\begin{array}{l}\text { Structured activity } \\
\text { without childcare at } \\
24 \text { months }\end{array}$ & $\begin{array}{c}-2.37(-7.59 \text { to } 2.84) \\
(-0.14)\end{array}$ & $0.37(0.48)$ & $25(18.1)$ & $40(30.3)$ & -3.94 (-9.69 to 1.82$)$ & $0.18(0.26)$ \\
\hline \multicolumn{7}{|c|}{ Secondary time use outcomes } \\
\hline $\begin{array}{l}\text { Constructive economic } \\
\text { activity at } 9 \text { months }\end{array}$ & $\begin{array}{c}-1.14(-4.74 \text { to } 2.45) \\
(-0.06)\end{array}$ & $0.53(0.95)$ & $12(8.7)$ & $17(12.9)$ & $-1.36(-5.14$ to 2.42$)$ & $0.48(0.80)$ \\
\hline $\begin{array}{l}\text { Constructive economic } \\
\text { activity at } 15 \text { months }\end{array}$ & $\begin{array}{c}-4.44(-9.88 \text { to } 1.01) \\
(-0.21)\end{array}$ & $0.11(0.085)$ & $11(8.0)$ & $24(18.2)$ & $-6.92(-12.72$ to -1.12$)$ & $0.20(0.049)$ \\
\hline $\begin{array}{l}\text { Constructive economic } \\
\text { activity at } 24 \text { months }\end{array}$ & $\begin{array}{c}-8.34(-14.41 \text { to } \\
-2.27),(-0.38)\end{array}$ & $0.01(0.038)$ & $25(18.1)$ & $40(30.3)$ & $-7.97(-15.02$ to -0.93$)$ & $0.03(0.073)$ \\
\hline
\end{tabular}

Overall, 235 (87\%) participants provided data for the primary outcome of weekly hours in structured activity at 15 months postrandomisation, with data missing for $13 \%$ of participants $(n=11$ in the SRT plus ESC group, $n=24$ in the ESC alone group; Fig. 1 and Table 2). The primary ITT analysis provided no evidence for the superiority of SRT plus ESC over ESC alone in improving structured activity or constructive economic activity (Table 3). Interaction terms for ARMS status (in an at risk mental state for psychosis versus not) and social disability (low versus very low functioning) were non-significant. There was no evidence for the superiority of SRT plus ESC over ESC alone for structured activity at 9 or 24 months post-randomisation (Table 3). At 24 months, there was weak evidence of greater structured activity in the ESC alone group (Table 3). The per protocol analysis was consistent with the ITT analysis at all time points (Table 3). General linear models did not provide any evidence for the superiority of SRT over ESC at any assessment point in terms of transition to psychosis, attenuated psychotic symptoms, general mental health or other symptoms (Table 4). There was no evidence of the superiority of SRT for negative symptoms or rates of diagnosable mood, eating or somatoform disorders (supplementary information, available at https://doi.org/ 10.1192/bjp.2021.206). It is notable that in both trial arms, significant improvements were made from baseline to follow-up in time spent in structured activity, reduction in depression and anxiety symptoms and diagnoses, and improvements in other symptoms and markers of psychological well-being.

Primary outcome missing data was $11 \%$ at 9 months and $24 \%$ at 24 months post-randomisation. Missing data was greater for secondary outcomes across time points (Table 2). Moreover, there was evident differential missingness and a bias towards greater attrition in the ESC alone arm throughout the trial. Missing data modelling was performed using full information maximum likelihood and multiple imputation approaches. The missing data analysis was consistent with the ITT analysis and provided no evidence for the superiority of SRT plus ESC compared with ESC alone on any outcome.

There were 84 adverse events and 83 serious adverse events during the trial, affecting 59 and 42 individuals respectively. More serious adverse events, for example medication overdose, were reported by SRT plus ESC participants $(n=53)$ compared with ESC participants $(n=30)$. However, event frequencies became very similar when adjusting for only those reported at trial assessments and excluding those reported to SRT therapists: (SRT plus ESC: $n=36$; ESC alone: $n=30$ ). No events were attributable to the trial.

For health economic outcomes, the mean total intervention cost per SRT participant was $£ 3268.94$ (SRT plus ESC was estimated at $£ 5927.73)$ versus $£ 2420.61$ for ESC alone. The mean incremental cost for SRT was estimated to be $£ 3910.59$ (range: $£ 2708.32$ 5112.86) compared with ESC, with a QALY gain of 0.001 (range: -0.099 to 0.10 ). Therefore, SRT was not estimated to be cost-effective.

\section{Discussion}

The aim of this trial was to determine the clinical and cost-effectiveness of adding social recovery therapy (SRT) to enhanced standard care (ESC), compared with ESC alone, for young people with social disability and severe and complex mental health problems. We found no evidence of the superiority of SRT as an adjunct to ESC in the primary outcome of weekly hours in structured activity at the primary 15-month end-point or at 9 or 24 months post-randomisation. We found no evidence that SRT was superior to ESC in secondary or other outcomes at any time point. SRT was not estimated to be cost-effective.

On some outcomes, including negative symptoms at 15 months and structured activity at 24 months post-randomisation, there were 
Table 4 Pre-specified secondary and other outcome analysis using intention-to-treat population

\begin{tabular}{|c|c|c|c|c|}
\hline \multirow[b]{2}{*}{ Outcome and time point ${ }^{\mathrm{a}}$} & \multicolumn{2}{|c|}{ Intention to treat population } & \multicolumn{2}{|c|}{ Missing data } \\
\hline & Effect size $(95 \% \mathrm{Cl})$ & $\begin{array}{l}\text { Intervention } v \text {. control, } \\
\text { log-transformed } P\end{array}$ & $\begin{array}{c}\mathrm{SRT}+\mathrm{ESC} \\
(n=138) n(\%)\end{array}$ & $\begin{array}{c}\text { ESC alone } \\
(n=132) n(\%)\end{array}$ \\
\hline \multicolumn{5}{|l|}{ Secondary outcomes } \\
\hline Transition to psychosis at 9 months ${ }^{\mathrm{b}}$ & 1.30 (0.49 to 3.44$)$ & 0.59 & $16(11.6)$ & $25(18.9)$ \\
\hline Transition to psychosis at 15 months ${ }^{b}$ & $7.33(0.71$ to 76.25$)$ & 0.10 & $30(21.7)$ & $44(33.3)$ \\
\hline Transition to psychosis at 24 months $^{b}$ & $1.29(0.20$ to 8.19$)$ & 0.79 & $40(29.0)$ & $51(38.6)$ \\
\hline CAARMS symptom severity at 9 months & $2.26(-1.92$ to 6.43$),(0.06)$ & $0.29(0.40)$ & $21(15.2)$ & $28(21.2)$ \\
\hline CAARMS symptom severity at 15 months & $0.29(-4.35$ to 4.94$),(-0.05)$ & 0.90 & $23(16.7)$ & $42(31.8)$ \\
\hline CAARMS symptom severity at 24 months & 1.45 (-5.54 to 8.44$),(0.01)$ & 0.68 & $79(57.2)$ & $86(65.2)$ \\
\hline CAARMS symptom distress at 9 months & $2.11(-3.73$ to 7.95$),(0.11)$ & 0.48 & $27(19.6)$ & $28(21.2)$ \\
\hline CAARMS symptom distress at 15 months & $4.09(-3.52$ to 11.70$),(0.14)$ & 0.29 & $34(24.6)$ & $40(30.3)$ \\
\hline CAARMS symptom distress at 24 months & 11.64 (1.29 to 22.00$),(0.44)$ & 0.03 & $80(58.0)$ & $85(64.4)$ \\
\hline Current major depressive episode at 9 months ${ }^{c}$ & 0.71 (0.46 to 1.11$)$ & 0.18 & $13(9.4)$ & $19(14.4)$ \\
\hline Current major depressive episode at 15 months ${ }^{c}$ & 1.11 (0.72 to 1.72$)$ & 0.65 & $16(11.6)$ & $31(23.5)$ \\
\hline Current major depressive episode at 24 months ${ }^{c}$ & 1.04 (0.61 to 1.79$)$ & 1.00 & $32(23.2)$ & $49(37.1)$ \\
\hline Major depressive disorder at 9 months ${ }^{c}$ & $1.07(0.83$ to 1.37$)$ & 0.70 & $13(9.4)$ & $21(15.9)$ \\
\hline Major depressive disorder at 15 months $^{c}$ & $1.13(0.84$ to 1.51$)$ & 0.50 & $16(11.6)$ & $30(22.7)$ \\
\hline Major depressive disorder at 24 months $^{c}$ & 1.26 (0.87 to 1.83$)$ & 0.23 & $32(23.2)$ & $49(37.1)$ \\
\hline Current social phobia at 9 months ${ }^{c}$ & 0.75 (0.49 to 1.15$)$ & 0.23 & $7(5.1)$ & $9(6.8)$ \\
\hline Current social phobia at 15 months $^{c}$ & 1.33 (0.84 to 2.11$)$ & 0.30 & $8(5.8)$ & $13(9.8)$ \\
\hline Current social phobia at 24 months ${ }^{c}$ & 1.63 (0.99 to 2.67$)$ & 0.05 & $16(11.6)$ & $18(13.6)$ \\
\hline Current generalised anxiety disorder at 9 months ${ }^{c}$ & 0.75 (0.41 to 1.37$)$ & 0.46 & $3(2.2)$ & $8(6.1)$ \\
\hline Current generalised anxiety disorder at 15 months $^{c}$ & 1.34 (0.69 to 2.61$)$ & 0.44 & $4(2.9)$ & $11(8.3)$ \\
\hline Current generalised anxiety disorder at 24 months $^{c}$ & 1.14 (0.63 to 2.06$)$ & 0.79 & $9(6.5)$ & $18(13.6)$ \\
\hline BDI-II at 9 months & $-1.28(-4.83$ to 2.26$),(-0.09)$ & 0.48 & $24(17.4)$ & $32(24.2)$ \\
\hline BDI-II at 15 months & $-0.32(-4.06$ to 3.42$),(-0.01)$ & 0.87 & $25(18.1)$ & $38(28.8)$ \\
\hline BDI-II at 24 months & $1.93(-2.22$ to 6.08$),(0.03)$ & 0.36 & $41(29.7)$ & $51(38.6)$ \\
\hline SIAS at 9 months & $-2.56(-6.13$ to 1.01$),(0.01)$ & 0.16 & $23(16.7)$ & $29(22.0)$ \\
\hline SIAS at 15 months & -0.45 (-4.84 to 3.95$),(0.05)$ & 0.84 & $23(16.7)$ & $38(28.8)$ \\
\hline SIAS at 24 months & 1.48 (-3.50 to 6.45), (0.15) & 0.56 & $39(28.3)$ & $49(37.1)$ \\
\hline GAF at 9 months & $1.57(-2.12$ to 5.27$),(0.07)$ & 0.40 & $17(12.3)$ & $26(19.7)$ \\
\hline GAF at 15 months & $-0.93(-5.24$ to 3.38$),(-0.06)$ & 0.67 & $21(15.2)$ & $37(28.0)$ \\
\hline GAF at 24 months & $-2.87(-7.49$ to 1.76$),(-0.19)$ & 0.22 & $36(26.1)$ & $51(38.6)$ \\
\hline GAS at 9 months & 1.02 (-2.51 to 4.56$),(0.08)$ & 0.57 & $17(12.3)$ & $26(19.7)$ \\
\hline GAS at 15 months & $-1.72(-5.95$ to 2.51$),(-0.08)$ & 0.43 & $21(15.2)$ & $35(26.5)$ \\
\hline GAS at 24 months & $-3.63(-8.40$ to 1.14$),(-0.19)$ & 0.14 & $38(27.5)$ & $50(37.9)$ \\
\hline SOFAS at 9 months & $0.24(-3.37$ to 3.84$),(-0.11)$ & 0.90 & $17(12.3)$ & $26(19.7)$ \\
\hline SOFAS at 15 months & $0.60(-3.64$ to 4.84$),(-0.07)$ & 0.78 & $20(14.5)$ & $35(26.5)$ \\
\hline SOFAS at 24 months & $-2.05(-6.91$ to 2.81$),(-0.22)$ & 0.41 & $38(27.5)$ & $50(37.9)$ \\
\hline \multicolumn{5}{|l|}{ Other outcomes } \\
\hline BHS at 9 months & $-0.17(-1.70$ to 1.36$),(0.02)$ & 0.83 & $28(20.3)$ & $33(25.0)$ \\
\hline BHS at 15 months & $-0.17(-1.80$ to 1.47$),(0.00)$ & 0.84 & $22(15.9)$ & $39(29.5)$ \\
\hline BHS at 24 months & 1.40 (-0.34 to 3.14$),(0.29)$ & 0.12 & $40(29.0)$ & $52(39.4)$ \\
\hline AUDIT at 9 months & 0.52 (-0.67 to 1.71$),(0.03)$ & $0.39(0.92)$ & $23(16.7)$ & $29(22.0)$ \\
\hline AUDIT at 15 months & 0.63 (-0.69 to 1.95$),(0.02)$ & $0.35(0.46)$ & $22(15.9)$ & $35(26.5)$ \\
\hline AUDIT at 24 months & $0.24(-0.68$ to 1.16$),(-0.05)$ & $0.61(0.27)$ & $36(26.1)$ & $49(37.1)$ \\
\hline DUDIT at 9 months & $-0.71(-2.02$ to 0.61$),(-0.10)$ & $0.29(0.42)$ & $19(13.8)$ & $28(21.2)$ \\
\hline DUDIT at 15 months & $-1.05(-2.54$ to 0.45$),(-0.12)$ & $0.17(0.46)$ & $19(13.8)$ & $39(29.5)$ \\
\hline DUDIT at 24 months & $-1.36(-2.86$ to 0.14$),(-0.18)$ & $0.08(0.01)$ & $34(24.6)$ & $47(35.6)$ \\
\hline \multicolumn{5}{|c|}{$\begin{array}{l}\text { SRT, social recovery therapy; ESC, enhanced standard care; CAARMS, Comprehensive Assessment of At Risk Mental States; BDI-II, Beck Depression Inventory II; SIAS, Social Interaction } \\
\text { Anxiety Scale; } \text {;AF, Global Assessment of functioning; GAS, Global Assessment of Symptoms; SOFAS, Social and Occupational Functioning Scale; BHS, Beck Hopelessness Scale; AUDIT, } \\
\text { Alcohol Use Disorders Identification Test; DUDIT, Drug Use Disorders Identification Test. } \\
\text { All other effect sizes are given as adjusted mean differences, with Cohen's } d \text { addditionally provided in parentheses. } \\
\text { a. The } 24 \text {-month assessment point was a late addition to the study protocol during the internal pilot at funder request and therefore reflects greater missing data. } \\
\text { b. Effect size: odds ratio. } \\
\text { c. Effect size: relative risk.. }\end{array}$} \\
\hline
\end{tabular}

mean differences in favour of ESC alone. However, these findings were inconsistent and rarely approached statistical significance. For other outcomes, including depression and anxiety diagnoses and drug use, there was weak evidence in favour of SRT plus ESC. Similarly, these results were inconsistent and not statistically significant.

There were very large gains made in both trial arms across primary and secondary outcomes. In both trial arms, there was an average gain of $11 \mathrm{~h}$ in structured activity at 15 months post-randomisation, which constitutes an almost threefold increase in clinically meaningful effect. There was more than $50 \%$ reduction in the prevalence of diagnosable depression, social phobia, panic disorder and agoraphobia in both groups, and large improvements in selfreported depression, social anxiety, hopelessness, schizotypy, and drug and alcohol disorders. This was surprising as only a minority of young people with emerging complex problems show reliable improvements following specialist youth mental health service provision. ${ }^{4}$ Current participants had greater symptomatology than those in this prior study and therefore more change may have been possible. However, current participants had been experiencing significant difficulties for at least 6 months and were accessing treatment as usual, so change without specialist intervention was unexpected. Nonetheless, our results support those of the IMPACT 
adolescent depression trial, ${ }^{27}$ in which optimised case management (i.e. including, as per this trial, psychological, employment and practical support) appeared equally effective with and without the addition of different psychotherapies. Moreover, recent studies demonstrate that some individuals at elevated risk of psychosis may show symptomatic and functional improvement over time. ${ }^{28}$ The present sample was heterogeneous with respect to symptoms and comorbidities and therefore may have included participants who naturally recovered, in addition to subgroups who responded well to shorter- or longer-term specialised interventions. ${ }^{29}$

Anticipating that standard care might be limited and inaccessible, we provided thorough assessment feedback and a best practice referral guide. The data show that comprehensive packages of evidence-based interventions of case management, psychological therapy, medication and support with employment were delivered to both arms of the trial in three youth mental health centres of excellence. It is important that the large gains made in social and clinical recovery occurred in the context of this very active and comprehensive provision, which constituted optimal care according to NICE guidelines. In the context of such comprehensive evidencebased care, what the trial perhaps shows is that providing further adjunctive specialised social recovery therapy is not required. Further research is warranted in understanding how benefits occurred and the factors associated with improvement. The implication for services is to try to actively engage, and deliver comprehensive assessment and treatment, to maximise outcomes for this often-neglected group.

\section{Participants' experience of the trial}

Our qualitative process evaluation found that SRT was experienced positively by the participants interviewed, that SRT was challenging but beneficial, and that it provided participants with a positive therapeutic relationship and specific tools needed to pursue social recovery goals. ${ }^{21}$ Participants in both arms described benefits of trial involvement, experiencing the assessment process as facilitating self-reflection and highly valuing contact with warm, empathic assessors. ${ }^{20}$ SRT was contrasted with standard care, which participants described as too limited. ${ }^{21}$ It is notable that trial assessment procedures were experienced by participants as facilitating hope $^{20,21}$ and may have increased standard treatment engagement alongside our deliberate attempts to encourage providers to offer comprehensive treatment packages. Our previous SUPEREDEN3 trial $^{19}$ found that SRT as an adjunct to EIP was superior in improving social and clinical recovery. Thus, adjunctive SRT may become necessary and effective only following transition to psychosis and, moreover, in a group characterised by greater homogeneity, i.e. male gender, and persistent and severe symptoms and social disability despite prior provision of specialist mental health services, such as in the SUPEREDEN3 trial. ${ }^{19}$ This is in keeping with the plasticity of the adolescent brain, which while conferring heightened vulnerability also provides greatest receptivity and malleability to resilience-enhancing factors, meaning that adolescence is a period of greatest therapeutic gains. ${ }^{30}$ Further research is needed to disentangle the characteristics that distinguish persistence of social disability in psychosis from what appears from this trial to be greater responsivity to treatment in the absence of psychosis but in the presence of mental health problems of equivalent severity and complexity.

\section{Limitations}

As evident at baseline, trial participants were extremely withdrawn and severely symptomatic. The inclusion of this group in an intervention trial was challenging. We made considerable efforts to engage and retain participants but despite this, there was marked missing data, with greater disengagement in the control arm. There was an overrepresentation of males in the SRT plus ESC arm. Sex was not a stratifier, nor was any related analysis planned a priori, and the sex imbalance might have biased estimates. Nonetheless, the primary outcome analysis was regarded as appropriate and within pre-specified limits against bias, and alternative missing data models, full information maximum likelihood and multiple imputation were used to support this.

\section{Future research and clinical implications}

Future research should aim to further explore the factors associated with youth mental health problems that are persistent and resistant to standard treatment, ${ }^{4,5}$ and the characteristics and mechanisms associated with good response to existing intervention packages. Future research should identify how to optimise standard care depending on need and how to implement optimal combinations of evidence-based interventions for young people with complex problems. ${ }^{4,5}$ This may involve the consideration of stepped-care approaches to identify young people who are more treatment-resistant and require longer-term specialist social recovery intervention. ${ }^{4,5}$ Existing research suggests that young people with severe and complex problems struggle to access interventions., However, this study shows that, even with very severe social disability and complex mental health problems, young people can make very significant improvements if comprehensive packages of evidence-based care are provided. Such packages include detailed engagement and assessment, and access to psychological therapies and vocational support, alongside medical and social interventions. The gains obtained by such provision have been shown to be large in this study, and in this context, the addition of specialised social recovery therapy does not provide superior social or clinical recovery, nor is it cost-effective. The message of this trial is to recommend equitable provision of optimised evidence-based care packages for clinical and social recovery within services that are appropriate and accessible for young people. Participants in the trial's process evaluation emphasised the importance of motivation and self-agency in social recovery ${ }^{20,21}$ and delivering this care to young people in the context of hopefulness appears essential.

Clio Berry $\mathbb{D}$, School of Psychology, University of Sussex, Brighton, UK; and Research \& Development, Sussex Partnership NHS Foundation Trust, Brighton, UK; and Primary Care and Public Health, Brighton and Sussex Medical School, Brighton, UK;

Joanne Hodgekins, Norwich Medical School, University of East Anglia, Norwich, UK; and Research \& Development, Norfolk and Suffolk NHS Foundation Trust, Norwich, UK;

Paul French (D), Manchester Metropolitan University, Faculty of Health, Psychology and Social Care, UK; and Pennine Care Mental Health NHS Foundation Trust, Ashton-underLyne, UK; Tim Clarke, Research \& Development, Norfolk and Suffolk NHS Foundation Trust, Norwich, UK; Lee Shepstone, Norwich Clinical Trials Unit, University of East Anglia, Norwich, UK; Garry Barton, Norwich Clinical Trials Unit, University of East Anglia, Norwich, UK; Robin Banerjee, School of Psychology, University of Sussex, Brighton, UK: Rory Byrne, Psychosis Research Unit, Greater Manchester Mental Health NHS

Foundation Trust, Manchester, UK; Rick Fraser, Research \& Development, Sussex

Foundation Trust, Manchester, UK; Rick Fraser, Research \& Development, Sussex
Partnership NHS Foundation Trust, Brighton, UK; Kelly Grant, Norwich Clinical Trials Unit, University of East Anglia, Norwich, UK; Kathryn Greenwood, School of Psychology, University of Sussex, Brighton, UK; and Research \& Development, Sussex Partnership NHS Foundation Trust, Brighton, UK; Caitlin Notley, Norwich Medical School, University of East Anglia, Norwich, UK; Sophie Parker, Psychosis Research Unit, Greater Manchester Mental Health NHS Foundation Trust, Manchester, UK; Jon Wilson (D), Research \& Development, Norfolk and Suffolk NHS Foundation Trust, Norwich, UK; Alison R. Yung, Centre for Youth Mental Health, University of Melbourne, Australia; and School of Health Sciences, University of Manchester, UK; David Fowler, School of Psychology, University of Sussex, Brighton, UK; and Research \& Development, Sussex Partnership NHS Foundation Trust, Brighton, UK

Correspondence: Clio Berry. Email: c.berry@bsms.ac.uk

First received 23 Apr 2021, final revision 18 Oct 2021, accepted 30 Nov 2021

\section{Supplementary material}

Supplementary material is available online at https://doi.org/10.1192/bjp.2021.206. 


\section{Data availability}

The data that support the findings of this study are available from the corresponding author on reasonable request.

\section{Acknowledgements}

We are very grateful to the young people who participated in the trial and all family members, friends, referring services, clinicians and other people who supported their involvement. We thank the PRODIGY Advisory Team, Trial Steering Committee, and Data Monitoring and Ethics Committee members for their invaluable involvement and guidance throughout this trial. We wish to acknowledge the support of our National Institute for Health Research programme manager. We thank all staff in the sponsoring and hosting organisations for supporting the project. We are grateful to Norwich Clinical Trials Unit for their support with data management, statistical and health economic analysis. Finally, we thank all the PRODIGY therapists and research assistants for their enthusiasm and dedication to supporting participants in their involvement with PRODIGY.

\section{Author contributions}

D.F. and P.F. were co-chief investigators for this trial. T.C. and C.B. were pilot and extension phase trial managers respectively. C.B. wrote the first draft of this manuscript with D.F. and R.F. S.P., J.W. and A.R.Y. were co-investigators and contributed to the study design and delivery. All authors contributed to and approved this final manuscript. Multiple authors had access to the complete study data and the authors were fully responsible for the decision to publish this manuscript.

\section{Funding}

This project was funded by the National Institute for Health Research Health Technology Assessment programme $(10 / 104 / 51,10 / 104 / 501)$. The funding source had no role in the design of the study, data collection, analysis, interpretation nor in this manuscript.

\section{Declaration of interest}

\section{None.}

\section{References}

1 Fowler D, French P, Banerjee R, Barton G, Berry C, Byrne R, et al. Prevention and treatment of long-term social disability amongst young people with emerging severe mental illness with social recovery therapy (the PRODIGY Trial): study protocol for a randomised controlled trial. Trials 2017; 18: 315.

2 Fowler D, Hodgekins J, Arena K, Turner R, Lower R, Wheeler K, et al. Early detection and psychosocial intervention for young people who are at risk of developing long term socially disabling severe mental illness: should we give equal priority to functional recovery and complex emotional dysfunction as to psychotic symptom. Clin Neuropsychiatry 2010; 7: 63-72.

3 Fusar-Poli P, Davies C, Solmi M, Brondino N, De Micheli A, Kotlicka-Antczak M et al. Preventive treatments for psychosis: Umbrella review (just the evidence). Front Psychiatry 2019; 10: 764

4 Cross SPM, Scott JL, Hermens DF, Hickie IB. Variability in clinical outcomes for youths treated for subthreshold severe mental disorders at an early intervention service. Psychiatr Serv 2018; 69: 555-61.

5 Cross SPM, Hermens DF, Hickie IB. Treatment patterns and short-term outcomes in an early intervention youth mental health service. Early Interv Psychiatry 2016; 10: 88-97.

6 Burke AS, Shapero BG, Pelletier-Baldelli A, Deng WY, Nyer MB, Leathem L, et al. Rationale, methods, feasibility, and preliminary outcomes of a transdiagnostic prevention program for at-risk college students. Front Psychiatry 2020; 10 1030.

7 Kessler RC, Berglund P, Demler O, Jin R, Merikangas KR, Walters EE. Lifetime prevalence and age-of-onset distributions of DSM-IV disorders in the national comorbidity survey replication. Arch Gen Psychiatry 2005; 62: 593-602.

8 Cross SPM, Scott J, Hickie IB. Predicting early transition from sub-syndromal presentations to major mental disorders. BJPsych Open 2017; 3: 223-7.

9 Hodgekins J, French P, Birchwood M, Mugford M, Christopher R, Marshall M, et al. Comparing time use in individuals at different stages of psychosis and a non-clinical comparison group. Schizophr Res 2015; 161: 188-93.
10 Knapp M, King D, Healey A, Cicely T. Economic outcomes in adulthood and their associations with antisocial conduct, attention deficit and anxiety problems in childhood. J Ment Health Policy Econ 2011; 14: 137-47.

11 Department of Health. Future in Mind: Promoting, Protecting and Improving our Children and Young People's Mental Health and Wellbeing. Department of Health, 2015.

12 Devoe DJ, Farris MS, Townes P, Addington J. Interventions and social functioning in youth at risk of psychosis: a systematic review and meta-analysis. Early Interv Psychiatry 2019; 13: 169-80.

13 Laws KR, Darlington N, Kondel TK, McKenna PJ, Jauhar S. Cognitive behavioura therapy for schizophrenia - outcomes for functioning, distress and quality of life: a meta-analysis. BMC Psychol 2018; 6: 32

14 Scott J, Fowler D, McGorry P, Birchwood M, Killackey E, Christensen H, et al. Adolescents and young adults who are not in employment, education, or training. BMJ 2013; 347: f5270.

15 van Rijn RM, Carlier BE, Schuring M, Burdorf A. Work as treatment? The effectiveness of re-employment programmes for unemployed persons with severe mental health problems on health and quality of life: a systematic review and meta-analysis. Occup Environ Med 2016; 73: 275-9.

16 Hodgekins J, Birchwood M, Christopher R, Marshall M, Coker S, Everard L, et al Investigating trajectories of social recovery in individuals with first-episode psychosis: a latent class growth analysis. Br J Psychiatry 2015; 207: 536-43.

17 Fowler D, Hodgekins J, Berry C, Clarke T, Palmier-Claus J, Sacadura C, et al. Social recovery therapy: a treatment manual. Psychosis 2019: 261-72.

18 Barton GR, Hodgekins J, Mugford M, Jones PB, Croudace T, Fowler D. Cognitive behaviour therapy for improving social recovery in psychosis: cost-effectiveness analysis. Schizophr Res 2009; 112: 158-63.

19 Fowler D, Hodgekins J, French P, Marshall M, Freemantle N, McCrone P, et al. Social recovery therapy in combination with early intervention services for enhancement of social recovery in patients with first-episode psychosis (SUPEREDEN3): a single-blind, randomised controlled trial. Lancet Psychiatry 2018; 5: 41-50.

20 Notley C, Christopher R, Hodgekins J, Byrne R, French P, Fowler D. Participan views on involvement in a trial of social recovery cognitive-behavioural therapy. Br J Psychiatry 2015; 206: 122-7

21 Gee B, Notley C, Byrne R, Clarke T, Hodgekins J, French P, et al. Young people's experiences of social recovery cognitive behavioural therapy and treatment as usual in the PRODIGY trial. Early Interv Psychiatry 2018; 12: 879-85.

22 Hodgekins J, Fowler D. CBT and recovery from psychosis in the ISREP trial: mediating effects of hope and positive beliefs on activity. Psychiatr Serv 2010; 61: $321-4$

23 Lowen C, Hodgekins J, Pugh K, Berry C, Fitzsimmons M, French P, et al. Measuring adherence in social recovery therapy with people with first episode psychosis. Behav Cogn Psychother 2020; 48: 82-90.

24 Blackburn I-M, James IA, Milne DL, Baker C, Standart S, Garland A, et al. The Revised Cognitive Therapy Scale (CTS-R): psychometric properties. Behav Cogn Psychother 2001; 29: 431-46.

25 Short S. Review of the UK 2000 Time Use Survey. Office for National Statistics, 2006

26 Morrison AP, Stewart SLK, French P, Bentall RP, Birchwood M, Byrne R, et al. Early detection and intervention evaluation for people at high-risk of psychosis-2 (EDIE-2): trial rationale, design and baseline characteristics. Early Interv Psychiatry 2011; 5: 24-32.

27 Goodyer IM, Reynolds S, Barrett B, Byford S, Dubicka B, Hill J, et al. Cognitive behavioural therapy and short-term psychoanalytical psychotherapy versus a brief psychosocial intervention in adolescents with unipolar major depressive disorder (IMPACT): a multicentre, pragmatic, observer-blind, randomised controlled superiority trial. Lancet Psychiatry 2017; 4: 109-19.

28 Yung AR, Nelson B, Thompson A, Wood SJ. The psychosis threshold in ultra high risk (prodromal) research: is it valid? Schizophr Res 2010; 120: 1-6.

29 Hickie IB, Scott EM, Hermens DF, Naismith SL, Guastella AJ, Kaur M, et al. Applying clinical staging to young people who present for mental health care. Early Interv Psychiatry 2013; 7: 31-43.

30 Malhi GS, Das P, Bell E, Mattingly G, Mannie Z. Modelling resilience in adolescence and adversity: a novel framework to inform research and practice. Transl Psychiatry 2019; 9: 316 